\title{
Raymond Castaing's Ion Microscope and Secondary Ion Mass Spectrometry
}

\author{
Peter Williams \\ Department of Chemistry \& Biochemistry, Arizona State University, Tempe, AZ 85287
}

In the late 1950's Raymond Castaing had seen his electron microprobe become an essential tool of microanalysis, and he turned his attention to a potential new method of analyzing solids, using the then little-explored phenomenon of sputtered ion emission to generate analytical signals that consisted literally of the atoms of the sample, ejected and ionized by impact of energetic primary ions on the sample surface. The existence of this ion emission phenomenon had been known since the pioneering work of J.J. Thompson around 1910, but little fundamental study had been carried out and the theoretical basis of the phenomenon was not understood. Castaing was fortunate to have as a new graduate student a young Georges Slodzian, to whom he gave the project of developing a new microanalytical tool that used a mass spectrometer to generate images using secondary ions sputtered from surfaces - Secondary Ion Mass Spectrometry (SIMS). Although the physics of ion microbeam generation was reasonably straightforward even then, Slodzian recalls that Castaing refused to take this obvious route and instead noted that, unlike X-rays but like electrons, ions could be focused by electrostatic and magnetic lenses so that there was the possibility to build a directimaging ion microscope rather than a microprobe. Such emission microscopes, for secondary electrons, had already been demonstrated before World War II by Mollenstedt and others (the technology has been revived in recent decades by Ernst Bauer). It seems that Castaing chose this path primarily for its elegance, and he described it to Slodzian as "a pleasant exercise in optics". One wonders what the young graduate student, who must certainly have realized that a microprobe would be simpler to build, thought of this "pleasant exercise" that was clearly going to lengthen his graduate student career.

There were numerous hurdles to overcome to build such a device, particularly in what was still fairly early post-war France. One of the first was to determine if the ions were indeed even formed at the sample surface. A simple comparison of sputtered ion velocities and the much greater velocity of electrons at the Fermi level suggested that the slow-moving ions should be efficiently neutralized by electrons tunneling from the surface, and there seemed a high probability that the observed signals might actually be formed in gas-phase collisions of sputtered atoms with the incoming primary ions far from the surface - and out of range of neutralization. Indeed this process was later shown to occur, but if it were dominant it would be a death knell for emission microscope imaging, which relies on the ions all emerging from a common equipotential surface. So Slodzian's first task was to demonstrate using a retarding potential approach that the majority of sputtered ions were indeed formed at the surface. (It is only in the last few years that we are beginning to understand how that can happen - it depends on the electrons in the impact site becoming excited during the brief instant required for the sputtered ions to depart.)

Electrostatic lenses to focus charged particles were by then quite well developed for electron microscope and microprobe technology, but ion microscopy would require also a mass spectrometer through which ions could be passed stigmatically without losing the image registration with the sample. To do this Castaing and Slodzian chose to use the focusing properties of the fringe fields of a magnetic sector. At the time, most mass spectrometer designers considered fringe fields an 
annoying nuisance to be limited and corrected for, rather than an ion-optical element. But Castaing was intimately familiar with pre- and post-war developments in electron optics, one of which had been the realization that non-normal entry of charged particles into a sector magnetic field allowed the horizontal component of the fringe field directed perpendicular to the particle trajectory to exert a vertical focusing action. Coupled with the already well-known first-order direction-focusing property of a deflection field, the fringe field focusing could bring ions diverging both horizontally and vertically to a common focal point.

The first ion microscope, built by Slodzian as a graduate student and described in his $\mathrm{PhD}$ thesis, was both a tour de force and a work of art. The beautiful ion images Slodzian obtained with that prototype design already achieved the theoretical resolution limit, around $0.5 \mu \mathrm{m}$, imposed by aberrations arising from the angle and energy spread of the sputtered ions. Despite almost 50 years of development by Cameca, the French company founded on Castaing's electron microprobe technology that licensed the ion microscope and has gone on to build something close to a world monopoly on imaging SIMS technology, even today it is not possible to surpass the ion microscope image resolution achieved by the young Georges Slodzian for his $\mathrm{PhD}$ defence.

Below a spatial resolution on the order of a few $\mu \mathrm{m}$ it starts to become advantageous to return finally to microprobe imaging. The reason is that apertures in the ion microscope must continually be decreased to reject off-axis ions and minimize spherical aberrations, sacrificing transmission, whereas, if the ions are generated with a microbeam, the mass spectrometer transmission is decoupled from the spatial resolution and can remain high. At the same time, with the first generation of commercial instruments - not just the Cameca ion microscope but also a microprobe designed by Helmut Liebl and built by Applied Research Laboratories (ARL) in the USA - it quickly became apparent that a significant limitation was going to be mass interferences arising from cluster ions that were found to be sputtered in quite high abundance from most samples. Thus a high mass resolving power was required to obtain clean signals, and for this again a microprobe design was preferable because ions ejected from a small spot could be introduced into the mass spectrometer with close to zero angular spread, greatly easing the problem of achieving high mass resolving power. Beginning in the 1980s Slodzian, in the prime of his long career as a Professor of Physics at the University of Paris, set out to design his last and best instrument, one that would achieve the highest possible spatial resolution, coupled with the highest ionization efficiency, mass spectrometer transmission and overall analytical efficiency. The result was the instrument now marketed by Cameca as the NanoSIMS. This instrument uses a $\mathrm{Cs}^{+}$primary ion beam to give high negative ionization probabilities, approaching $100 \%$ for the most electronegative elements. The beam is focused to a sub-50 nm spot size ( $25 \mathrm{~nm}$ has been demonstrated) using an objective lens with an extremely short working distance, and the secondary ions are extracted with very high efficiency through the same lens. Tiny probe sizes mean that we are sampling tiny numbers of atoms, so efficiency is crucial and the mass spectrometer has a $\sim 60 \mathrm{~cm}$ long focal plane, over the entire length of which mass resolving power up to 9,000 is specified; up to seven chosen ion species can be detected simultaneously along this plane with low background ion counting electron multipliers positioned using stepping motors. To date (February 2011) 24 such instruments have been delivered worldwide (the $24^{\text {th }}$ to the author); this instrument is beginning to impact dramatically fields as diverse as cosmochemistry, geochemistry, biochemistry and biomedical research - a development that surely would have gladdened the heart of Professor Raymond Castaing. 